HEPHY-PUB 968/16

UWThPh-2016-12

October 2016

\title{
INSTANTANEOUS BETHE-SALPETER VIEW OF GOLDSTONE-TYPE PSEUDOSCALAR MESONS
}

\author{
Wolfgang LUCHA* \\ Institute for High Energy Physics, \\ Austrian Academy of Sciences, \\ Nikolsdorfergasse 18, A-1050 Vienna, Austria \\ Franz F. SCHÖBERL ${ }^{\dagger}$ \\ Faculty of Physics, University of Vienna, \\ Boltzmanngasse 5, A-1090 Vienna, Austria
}

\begin{abstract}
Describing the lightest pseudoscalar mesons as bound states of quark and antiquark within the framework of an instantaneous Bethe-Salpeter formalism constructed such as to retain (in contrast to Salpeter's equation) as much information on the relativistic effects provided by the full quark propagator as conceivable allows for a surprisingly simple implementation of their near masslessness mandatory for their interpretability as pseudo-Goldstone bosons related to the spontaneous breaking of the chiral symmetries of quantum chromodynamics.
\end{abstract}

PACS numbers: 11.10.St, 03.65.Ge, 03.65.Pm

* E-mail address: wolfgang.lucha@oeaw.ac.at

$\dagger$ E-mail address: franz.schoeberl@univie.ac.at 


\section{Introduction}

The pseudo-Goldstone bosons of the spontaneously and to a minor degree explicitly broken chiral symmetries of quantum chromodynamics (QCD), the quantum field theory encoding all strong interactions in elementary particle theory, are commonly identified with the light pseudoscalar mesons, the pions and kaons. As quark-antiquark bound states, these may be described, in principle, by the Bethe-Salpeter formalism [1]; in practice, such attempt faces several well-known difficulties. In view of this, we devised [2] a three-dimensional reduction (the most famous of this kind still is Salpeter's equation [3]) of the Bethe-Salpeter equation by considering the instantaneous limit of the latter but allowing for approximations only to the extent definitely necessary for the analytic formulation of our bound-state equation [2].

In order to elucidate the relationship between the pseudo-Goldstone bosons of QCD, on the one hand, and their QCD-based description within the (instantaneous) Bethe-Salpeter framework, on the other hand, we recently devised an adequate inversion procedure [4] and applied it [5] to the maybe conceptually simplest three-dimensional reduction, the Salpeter equation, for which we analysed, in turn, the impact of implementing the proper ultraviolet asymptotic behaviour [6], of constraints imposed by axiomatic quantum field theory [7], and of exploiting available numerical knowledge [8] of, in all three cases, the Goldstone solution. Here, we generalize these Goldstone-boson analyses to the bound-state equation of Ref. [2].

The outline of this paper is as follows. In Sec. 2, we briefly sketch the cornerstones of the instantaneous Bethe-Salpeter formalism developed in Ref. [2], with particular focus on the physical scenario relevant here. In Sec. 3, we generalize the inversion technique of Ref. [4] to the bound-state equation derived in Ref. [2]. In Sec. 4, aided by a Ward-Takahashi identity, we relate the Goldstonic Bethe-Salpeter solution to the shape of the full quark propagator. In Sec. 5, we derive the potential entering in our instantaneous interaction kernel. In Sec. 6, we summarize our findings. (For convenience, we use natural units throughout: $\hbar=c=1$.)

\section{Instantaneous Limit of Bethe-Salpeter Framework}

\subsection{Dressed-propagator instantaneous Bethe-Salpeter equation}

Within the Poincaré-covariant Bethe-Salpeter formalism [1], a bound state of two particles of momenta $p_{1,2}$ is characterized by a momentum-dependent Bethe-Salpeter amplitude, $\Phi$. The basic idea behind our approach [2] is to describe fermion-antifermion bound states, for total momentum $P$ and relative momentum $p$ of their constituents and mass eigenvalues $\widehat{M}$ defined by $P^{2}=\widehat{M}^{2}$, by a three-dimensional reduction of the homogeneous Bethe-Salpeter equation which, nevertheless, retains relativistic effects to the utmost possible extent. Such static reduction becomes possible if assuming all underlying interactions to be independent of the time components of the relative fermion momenta in the center-of-momentum frame. The sole obstacles to this are then the full fermion propagators entering in the bound-state equation. Any fermion propagator $S(p)$ is defined by two Lorentz-scalar functions that may be chosen to be a mass function, $M\left(p^{2}\right)$, and a wave-function renormalization factor, $Z\left(p^{2}\right)$ :

$$
S_{i}(p)=\frac{\mathrm{i} Z_{i}\left(p^{2}\right)}{\not p-M_{i}\left(p^{2}\right)+\mathrm{i} \varepsilon}, \quad \not p \equiv p^{\mu} \gamma_{\mu}, \quad \varepsilon \downarrow 0, \quad i=1,2 .
$$

As a remedy, we approximate the propagators $S_{i}(p)$ by retaining only terms linear in $p_{0}$ : an integration with respect to $p_{0}$ then gives a bound-state equation for the Salpeter amplitude 


$$
\phi(\boldsymbol{p}) \equiv \frac{1}{2 \pi} \int \mathrm{d} p_{0} \Phi(p, P)
$$

The considerations of Ref. [2] resulted in the instantaneous Bethe-Salpeter equation for fermion-antifermion bound states with full propagators of the bound-state constituents [2]

$$
\phi(\boldsymbol{p})=Z_{1}\left(\boldsymbol{p}_{1}^{2}\right) Z_{2}\left(\boldsymbol{p}_{2}^{2}\right)\left(\frac{\Lambda_{1}^{+}\left(\boldsymbol{p}_{1}\right) \gamma_{0} I(\boldsymbol{p}) \gamma_{0} \Lambda_{2}^{-}\left(\boldsymbol{p}_{2}\right)}{P_{0}-E_{1}\left(\boldsymbol{p}_{1}\right)-E_{2}\left(\boldsymbol{p}_{2}\right)}-\frac{\Lambda_{1}^{-}\left(\boldsymbol{p}_{1}\right) \gamma_{0} I(\boldsymbol{p}) \gamma_{0} \Lambda_{2}^{+}\left(\boldsymbol{p}_{2}\right)}{P_{0}+E_{1}\left(\boldsymbol{p}_{1}\right)+E_{2}\left(\boldsymbol{p}_{2}\right)}\right),
$$

wherein $E_{i}(\boldsymbol{p})$ and $\Lambda_{i}^{ \pm}(\boldsymbol{p})$ are the one-particle free energies and energy projection operators

$$
E_{i}(\boldsymbol{p}) \equiv \sqrt{\boldsymbol{p}^{2}+M_{i}^{2}\left(\boldsymbol{p}^{2}\right)}, \quad \Lambda_{i}^{ \pm}(\boldsymbol{p}) \equiv \frac{E_{i}(\boldsymbol{p}) \pm \gamma_{0}\left[\boldsymbol{\gamma} \cdot \boldsymbol{p}+M_{i}\left(\boldsymbol{p}^{2}\right)\right]}{2 E_{i}(\boldsymbol{p})}, \quad i=1,2
$$

and all interactions experienced by the bound-state constituents are subsumed by the term

$$
I(\boldsymbol{p}) \equiv \frac{1}{(2 \pi)^{3}} \int \mathrm{d}^{3} q K(\boldsymbol{p}, \boldsymbol{q}) \phi(\boldsymbol{q})
$$

Of course, the center-of-momentum frame of the two-particle system under consideration is defined by $\boldsymbol{P}=0$, whence $P_{0}=\widehat{M}$ and $\boldsymbol{p}=\boldsymbol{p}_{1}=-\boldsymbol{p}_{2}$, and our bound-state equation reads

$$
\phi(\boldsymbol{p})=Z_{1}\left(\boldsymbol{p}^{2}\right) Z_{2}\left(\boldsymbol{p}^{2}\right)\left(\frac{\Lambda_{1}^{+}(\boldsymbol{p}) \gamma_{0} I(\boldsymbol{p}) \Lambda_{2}^{-}(\boldsymbol{p}) \gamma_{0}}{\widehat{M}-E_{1}(\boldsymbol{p})-E_{2}(\boldsymbol{p})}-\frac{\Lambda_{1}^{-}(\boldsymbol{p}) \gamma_{0} I(\boldsymbol{p}) \Lambda_{2}^{+}(\boldsymbol{p}) \gamma_{0}}{\widehat{M}+E_{1}(\boldsymbol{p})+E_{2}(\boldsymbol{p})}\right) .
$$

The particular projector structure of the right-hand side of our bound-state equation, somewhat symbolically given by $\Lambda_{1}^{+} \otimes \Lambda_{2}^{-}+\Lambda_{1}^{-} \otimes \Lambda_{2}^{+}$, allows for a generally valid observation. Multiplying Eqs. (1) or (3) from both left and right by appropriate energy projectors of the same sign cause their right-hand sides to vanish. Hence, all solutions $\phi(\boldsymbol{p})$ inevitably satisfy

$$
\Lambda_{1}^{+}\left(\boldsymbol{p}_{1}\right) \phi(\boldsymbol{p}) \Lambda_{2}^{+}\left(\boldsymbol{p}_{2}\right)=\Lambda_{1}^{-}\left(\boldsymbol{p}_{1}\right) \phi(\boldsymbol{p}) \Lambda_{2}^{-}\left(\boldsymbol{p}_{2}\right)=0 \text {. }
$$

The implications of retaining, in the three-dimensional reduction of the Bethe-Salpeter equation, relativistic effects as far as conceivable ${ }^{1}$ by use of Eq. (1) for the (semirelativistic) description [9] of hadrons as bound states of quarks [10] have been analysed in Refs. [11,12]: comparison with earlier studies of Salpeter's equation [13-16] revealed that the inclusion of full quark propagators exerts a substantial impact on the mass spectra of the bound states.

\subsection{Spin-singlet fermion-antifermion states: Salpeter amplitude}

Bearing in mind the fact that the actual targets of the present study, the pseudo-Goldstone bosons of QCD, are pseudoscalar mesons, let us recall ${ }^{2}$ the general Lorentz structure of the Salpeter amplitude for bound states of fermion and antifermion whose spins add up to zero.

The expansion of an arbitrary Salpeter amplitude over the basis generated by the Dirac matrices introduces 16 component functions. However, by the constraint (4) this number is cut down by a factor of two. Therefore, the most general solution $\phi(\boldsymbol{p})$ of Eqs. (1) or (3) can

\footnotetext{
${ }^{1}$ Upon the assumption that all bound-state constituents propagate freely with effective masses $m_{i}$, that is, in the free-propagator limit $M_{i}\left(\boldsymbol{p}^{2}\right) \rightarrow m_{i}$ and $Z_{i}\left(\boldsymbol{p}^{2}\right) \rightarrow 1$, constituting a still further step of simplifying approximation, the bound-state equation (3) reduces to — and, thus, generalizes — Salpeter's equation [3].

${ }^{2}$ The appropriate reasoning resembles the one used in, e.g., Refs. [17] for the case of Salpeter's equation.
} 
involve merely eight independent components. The latter differ in their response to discrete symmetry transformations: Out of these eight independent components, only two show the behaviour expected for the spin-singlet bound states we are interested in. Calling these two components $\varphi_{1,2}(\boldsymbol{p})$ and imposing flavour symmetry by requiring the fermion mass equality $M_{1}\left(\boldsymbol{p}^{2}\right)=M_{2}\left(\boldsymbol{p}^{2}\right)=M\left(\boldsymbol{p}^{2}\right)$, in the center-of-momentum frame of our fermion-antifermion system under consideration the general solution for spin-singlet states has to be of the form

$$
\phi(\boldsymbol{p})=\left[\varphi_{1}(\boldsymbol{p}) \frac{\gamma_{0}\left[\boldsymbol{\gamma} \cdot \boldsymbol{p}+M\left(\boldsymbol{p}^{2}\right)\right]}{E(\boldsymbol{p})}+\varphi_{2}(\boldsymbol{p})\right] \gamma_{5}, \quad E(\boldsymbol{p}) \equiv \sqrt{\boldsymbol{p}^{2}+M^{2}\left(\boldsymbol{p}^{2}\right)}
$$

Pseudoscalar states correspond to zero relative orbital angular momentum of the fermions.

\subsection{Flavour-, Fierz- and spherical-symmetry-enabled reductions}

Now, in order to convert our three-dimensional bound-state equation, given in Eq. (3) in its momentum-space representation, to a well-defined manageable inversion problem, we have to provide the particular specifications of the physical scenario under consideration. To this end, let us formulate a few simplifying assumptions emerging from symmetry requirements that are dictated by physics or, at least, may be justified on physical grounds. Our ultimate goal will be to characterize the effective interactions responsible for the formation of bound states in configuration-space representation by some central potential $V(r)$, where $r \equiv|\boldsymbol{x}|$.

1. Flavour symmetry implies equality both of all wave-function renormalization factors,

$$
Z_{1}\left(\boldsymbol{p}^{2}\right)=Z_{2}\left(\boldsymbol{p}^{2}\right)=Z\left(\boldsymbol{p}^{2}\right),
$$

and of all mass functions, with clear consequences for kinetic energies and projectors:

$$
M_{1}\left(\boldsymbol{p}^{2}\right)=M_{2}\left(\boldsymbol{p}^{2}\right)=M\left(\boldsymbol{p}^{2}\right), \quad E_{1}(\boldsymbol{p})=E_{2}(\boldsymbol{p})=E(\boldsymbol{p}), \quad \Lambda_{1}^{ \pm}(\boldsymbol{p})=\Lambda_{2}^{ \pm}(\boldsymbol{p})=\Lambda^{ \pm}(\boldsymbol{p}) .
$$

So, the flavour-symmetric limit of our full-propagator bound-state equation (3) reads

$$
\phi(\boldsymbol{p})=Z^{2}\left(\boldsymbol{p}^{2}\right)\left(\frac{\Lambda^{+}(\boldsymbol{p}) \gamma_{0} I(\boldsymbol{p}) \Lambda^{-}(\boldsymbol{p}) \gamma_{0}}{\widehat{M}-2 E(\boldsymbol{p})}-\frac{\Lambda^{-}(\boldsymbol{p}) \gamma_{0} I(\boldsymbol{p}) \Lambda^{+}(\boldsymbol{p}) \gamma_{0}}{\widehat{M}+2 E(\boldsymbol{p})}\right) .
$$

2. The integral kernel $K(\boldsymbol{p}, \boldsymbol{q})$ in the interaction term (2) subsumes both Lorentz nature - by generalized Dirac matrices $\Gamma_{1,2}$ - and momentum dependence - by associated Lorentz-scalar potential functions $V_{\Gamma}(\boldsymbol{p}, \boldsymbol{q})$ - of the effective interaction experienced by the bound-state constituents; for $\Gamma_{1}=\Gamma_{2}=\Gamma$, the action of $K(\boldsymbol{p}, \boldsymbol{q})$ on $\phi(\boldsymbol{p})$ reads

$$
K(\boldsymbol{p}, \boldsymbol{q}) \phi(\boldsymbol{q})=\sum_{\Gamma} V_{\Gamma}(\boldsymbol{p}, \boldsymbol{q}) \Gamma \phi(\boldsymbol{q}) \Gamma .
$$

Fierz symmetry is established by relying on the linear combination of tensor products

$$
\Gamma \otimes \Gamma=\frac{1}{2}\left(\gamma_{\mu} \otimes \gamma^{\mu}+\gamma_{5} \otimes \gamma_{5}-1 \otimes 1\right)
$$

3. The concurrence of the convolution nature of the interaction term $I(\boldsymbol{p})$ in Eq. (1) and the spherical symmetry of the momentum-space potential function $V(\boldsymbol{p}, \boldsymbol{q})$ related to the Lorentz structure (7), enforced by the requirement $V(\boldsymbol{p}, \boldsymbol{q})=V\left((\boldsymbol{p}-\boldsymbol{q})^{2}\right)$, implies that the Fourier transform of $V(\boldsymbol{p}, \boldsymbol{q})$ is a configuration-space central potential, $V(r)$. Clearly, this nice feature allows us to discard all dependence on the angular variables. 
Within the setting specified thereby, the dressed-propagator instantaneous Bethe-Salpeter equation (3) may be easily shown to reduce to the following system of coupled equations for the radial factors $\varphi_{1,2}(p), p \equiv|\boldsymbol{p}|$, of the independent components $\varphi_{1,2}(\boldsymbol{p})$ required by $\phi(\boldsymbol{p})$,

$$
\begin{aligned}
& 2 E(p) \varphi_{2}(p)+2 Z^{2}\left(p^{2}\right) \int_{0}^{\infty} \frac{\mathrm{d} q q^{2}}{(2 \pi)^{2}} V(p, q) \varphi_{2}(q)=\widehat{M} \varphi_{1}(p), \\
& 2 E(p) \varphi_{1}(p)=\widehat{M} \varphi_{2}(p),
\end{aligned}
$$

in apposite notation: $Z\left(\boldsymbol{p}^{2}\right)=Z\left(p^{2}\right), M\left(\boldsymbol{p}^{2}\right)=M\left(p^{2}\right)$ and $E(\boldsymbol{p})=E(p) \equiv \sqrt{p^{2}+M^{2}\left(p^{2}\right)}$. These relations pose an eigenvalue problem for the masses $\widehat{M} \equiv \sqrt{P^{2}}$ of the bound states in our focus of interest, wherein the interactions of the constituents enter under the disguise of

$$
V(p, q) \equiv \frac{8 \pi}{p q} \int_{0}^{\infty} \mathrm{d} r \sin (p r) \sin (q r) V(r), \quad q \equiv|\boldsymbol{q}| .
$$

The set of relations formed by Eqs. $(8 \mathrm{a}, 8 \mathrm{~b})$ might be considered as a kind of quintessence of our instantaneous bound-state formalism for the particular physical system defined before.

\section{Exact Inversive Instantaneous Goldstone Solutions}

Next, in order to tighten the noose around the precise way the strong interactions manifest, for the pseudo-Goldstone bosons of QCD, in our instantaneous description of bound states, let us explore the implications of the presumably most eye-catching property of particles of this kind, namely, the masslessness of Goldstone bosons requested by Goldstone's theorem.

Accordingly, we enumerate the not exorbitantly surprising implications of enforcing, by letting $\widehat{M}=0$, the Goldstone nature of each spin-singlet bound state obeying Eqs. (8a,8b):

1. For vanishing bound-state mass $\widehat{M}$, the two quintessence equations (8a,8b) decouple.

2. Equation (8b), being of algebraic nature, reduces to the demand $E(p) \varphi_{1}(p)=0$ that coerces one Salpeter component to vanish: $\varphi_{1}(\boldsymbol{p})=0$. Thus, each Salpeter amplitude (5) for massless spin-singlet fermion-antifermion bound states emerging as a solution to the flavour-symmetric limit (6) of the instantaneous bound-state equation (1) with Fierz-symmetric Dirac structure (7) of its interaction kernel assumes the simple form

$$
\phi(\boldsymbol{p})=\varphi_{2}(\boldsymbol{p}) \gamma_{5}
$$

3. Equation (8a), an integral equation reminiscent of the relevant interactions, becomes

$$
E(p) \varphi_{2}(p)+Z^{2}\left(p^{2}\right) \int_{0}^{\infty} \frac{\mathrm{d} q q^{2}}{(2 \pi)^{2}} V(p, q) \varphi_{2}(q)=0,
$$

which controls the surviving component $\varphi_{2}(\boldsymbol{p})$ and thus the Salpeter amplitude $\phi(\boldsymbol{p})$.

Needless to say, the envisaged extraction of the interaction potential $V(r)$ requires us to move, by means of a Fourier transformation, ${ }^{3}$ to configuration space. Anticipating that, for finite momenta $p$, the wave-function renormalization factor, $Z\left(p^{2}\right)$, will prove to be nonzero $\left[0 \supsetneqq Z\left(p^{2}\right) \leq 1\right]$, we divide Eq. (9) by $Z^{2}\left(p^{2}\right)$. By introducing the Fourier-Bessel transforms

\footnotetext{
${ }^{3}$ For functions of radial coordinates, Fourier transformations reduce to Fourier-Bessel transformations.
} 


$$
\begin{aligned}
& \varphi(r) \equiv \sqrt{\frac{2}{\pi}} \frac{1}{r} \int_{0}^{\infty} \mathrm{d} p p \sin (p r) \varphi_{2}(p), \\
& \widetilde{T}(r) \equiv \sqrt{\frac{2}{\pi}} \frac{1}{r} \int_{0}^{\infty} \mathrm{d} p p \sin (p r) \frac{E(p) \varphi_{2}(p)}{Z^{2}\left(p^{2}\right)}
\end{aligned}
$$

of the Salpeter component $\varphi_{2}(p)$ and of the ratio of the kinetic term $E(p) \varphi_{2}(p)$ and $Z^{2}\left(p^{2}\right)$, we arrive at the sought configuration-space representation of our bound-state equation (9):

$$
\widetilde{T}(r)+V(r) \varphi(r)=0 .
$$

From this, the Goldstone-promoting potential for the Lorentz structure (7) can be read off:

$$
V(r)=-\frac{\widetilde{T}(r)}{\varphi(r)}
$$

\section{Quark-Propagator-Defined Bethe-Salpeter Vertex}

From the aforegoing discussion, it should have become plain that the envisaged application of the inversion procedure sketched above requires as input the radial independent Salpeter component $\varphi_{2}(p)$ that defines the Salpeter amplitude $\phi(\boldsymbol{p})$ of the bound states under study. By definition, the latter quantity may be found from the Bethe-Salpeter amplitude $\Phi(p, P)$ of these very bound states, by an integration with respect to the time component $p_{0}$ of their constituents' relative four-momentum $p$. In the chiral limit, the (renormalized) axial-vector Ward-Takahashi identity of QCD turns out to relate the Bethe-Salpeter amplitude $\Phi(p, 0)$ for the flavour-nonsinglet pseudoscalar mesons to the (dressed) quark propagator $S(p)$ [18]. This latter two-point Green function, in turn, may be obtained as the solution to the quark Dyson-Schwinger equation (occasionally also found to be referred to as the gap equation). ${ }^{4}$

In Euclidean-space formulation, if skipping irrelevant overall factors and focusing to the dominant Dirac component, the relation between the Bethe-Salpeter amplitude $\Phi(\underline{k}, \underline{P})$ of a massless $\left(\underline{P}^{2}=-\widehat{M}^{2}=0\right)$ pseudoscalar meson in its center-of-momentum frame $(\underline{\boldsymbol{P}}=0)$ and the wave-function renormalization and mass functions $Z\left(\underline{k}^{2}\right)$ and $M\left(\underline{k}^{2}\right)$ governing the quark propagator $S(\underline{k})$ has been proven (cf. Sec. IV.B of Ref. [6]) to read, in the chiral limit,

$$
\Phi(\underline{k}, 0) \propto \frac{Z\left(\underline{k}^{2}\right) M\left(\underline{k}^{2}\right)}{\underline{k}^{2}+M^{2}\left(\underline{k}^{2}\right)} \underline{\gamma}_{5}+\text { subleading contributions . }
$$

The propagator functions are to be found by solving the quark Dyson-Schwinger equation.

The Dyson-Schwinger equation for the quark propagator is an element of the countably infinite hierarchy of the Dyson-Schwinger equations of QCD, that is, of a system of coupled integral equations that determines the infinity of $n$-point Green functions of the underlying quantum field theory. A manageable Dyson-Schwinger problem may only be formulated by appropriately decoupling, from this infinite tower, a finite subset of relations which include the Dyson-Schwinger equation governing the Green function of interest and continue to be coupled among each other. Such a truncation cuts the links between subset and remainder: necessary Green-function input has to be found by phenomenologically inspired modelling.

In order to put us in a position to take advantage, at least at a reasonable level of rigour, of the propagator-vertex interrelation (12) originating in the axial-vector Ward-Takahashi

\footnotetext{
${ }^{4}$ For good reasons, Dyson-Schwinger analyses are preferentially performed in Euclidean space; thus, we henceforth discriminate Euclidean-space coordinates and Minkowski-space ones by underlining the former.
} 
identity of QCD, the symmetry manifesting in form of this identity has to be respected also by the finite number of Dyson-Schwinger equations singled out by the favoured truncation. A class of models claimed to preserve the identity is the rainbow-ladder truncation scheme likewise imposed to quark Dyson-Schwinger equation and meson Bethe-Salpeter equation. Its renormalization-group-improved formulation is characterized by few generic properties:

1. The exact quark-gluon vertex function is substituted by its tree-level approximation.

2. The interaction kernel is cut down to its first perturbative term, one-gluon exchange.

3. The full gluon propagator entering the gap equation is approximated by its free form.

In order to compensate, at least in part, for thereby induced deficiencies, an effective strong fine-structure coupling is introduced that takes care of some phenomenological constraints:

- In the infrared $\underline{k}^{2} \rightarrow 0$, it gets enhanced, to satisfy the needs of the gluon propagator.

- In the ultraviolet $\underline{k}^{2} \rightarrow \infty$, its decay parallels that of the perturbative QCD coupling.

For our present analysis, we rely on the model proposed in Ref. [19], where the intended infrared enhancement of the effective strong coupling strength is accomplished without the introduction of a singular but integrable $\delta$ function in momentum space. Unfortunately, for the moment the solution to the Dyson-Schwinger equation for the quark propagator can be computed only in numerical form. Accordingly, we have to extract pointwise the behaviour of both propagator functions $M\left(\underline{k}^{2}\right)$ and $Z\left(\underline{k}^{2}\right)$ from their graphs available in the literature: Figure 1 shows the outcome of our simple interpolation of the results published in Ref. [20].

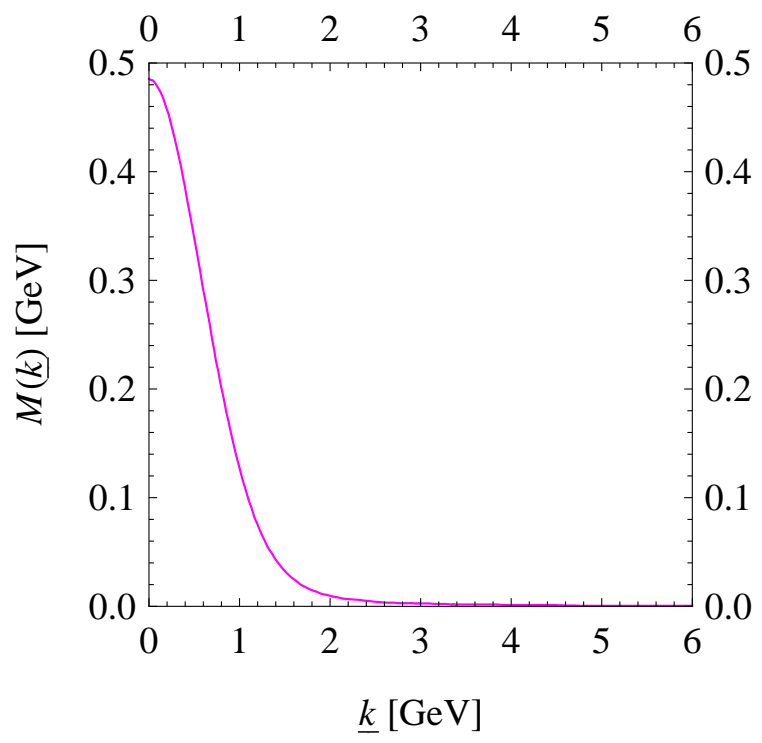

(a)

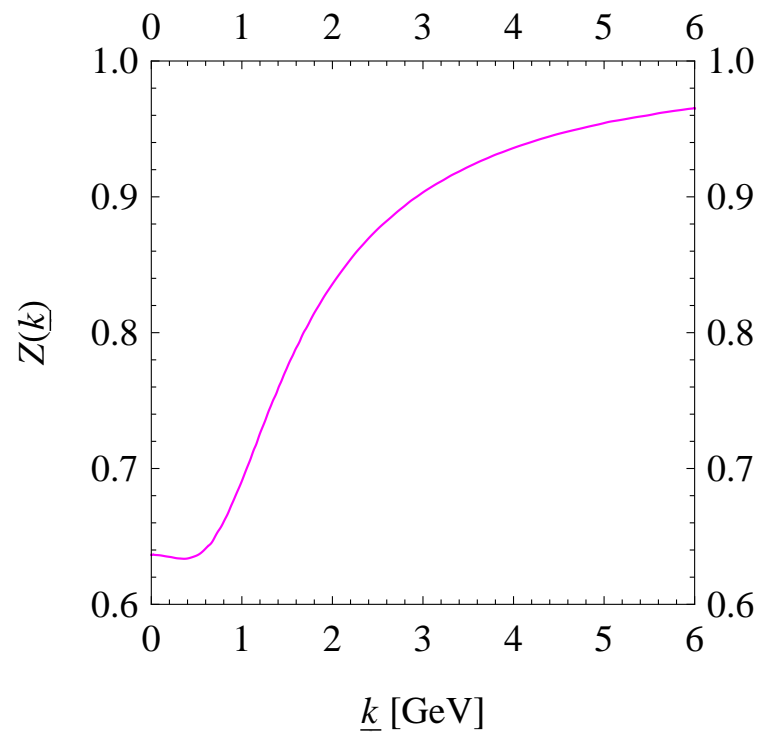

(b)

Figure 1: Chiral-limit solutions to the Dyson-Schwinger equation for the quark propagator $S(\underline{k})$ in the "renormalization-group-improved" rainbow-ladder model of Ref. [19]: (a) mass function $M(\underline{k})$ and (b) wave-function renormalization $Z(\underline{k})$ read off from Fig. 1 of Ref. [20]. 


\section{Goldstone-Boson-Generating Interaction Potential}

Equipped, in form of Fig. 1, with a solution for the full quark propagator, we convert it into the configuration-space potential $V(r)$. Being aware of the fact that we have at our disposal only information on $M(\underline{k})$ and $Z(\underline{k})$ from a limited interval of relative momenta $\underline{k}$, we make every effort to underpin our intermediate findings by pillars in form of analytic expressions.

First, we seek a parametrization of the propagator functions $M(\underline{k})$ and $Z(\underline{k})$ in terms of elementary functions. For the quark mass function, we rely on an ansatz resembling the one of our Ref. [8], with the numerical values of the five parameters $a, b, \gamma, c$, and $d$ as in Table 1 :

$$
M(\underline{k})=\frac{a}{\left(1+\underline{k}^{2} / b\right)^{\gamma}}+c \exp \left(-d \underline{k}^{2}\right) .
$$

For the quark wave-function renormalization, we try a shape complying with the behaviour $Z(\underline{k}) \rightarrow 1$ for $\underline{k} \rightarrow \infty$, finding for the six parameters $u, v, x, y, z$, and $\delta$ the values in Table 2 :

$$
Z(\underline{k})=u-\frac{v}{\left(1-x \underline{k}+y \underline{k}^{2}+z \underline{k}^{4}\right)^{\delta}} .
$$

Figure 2 confronts our parametrization of $M(\underline{k})$ and $Z(\underline{k})$ by the ansätze (13) and (14) with their interpolations given in Fig. 1. Beyond doubt, the agreement is more than satisfactory.

Table 1: Numerical values of the parameters in our ansatz (13) for the quark mass function.

\begin{tabular}{lccccc}
\hline \hline Parameter & $a[\mathrm{GeV}]$ & $b\left[\mathrm{GeV}^{2}\right]$ & $\gamma$ & $c[\mathrm{GeV}]$ & $d\left[\mathrm{GeV}^{-2}\right]$ \\
\hline Value & 0.112918 & 0.870237 & 1.53153 & 0.371719 & 1.39261 \\
\hline \hline
\end{tabular}

Next, by exploiting the Dyson-Schwinger-Bethe-Salpeter interplay (12), we determine the center-of-momentum Bethe-Salpeter amplitude (12) and from this, by integration with respect to the Euclidean-time coordinate $\underline{k}_{4}$, the sought radial component $\varphi_{2}(p), p \equiv|\boldsymbol{p}|$, of the three-dimensional Salpeter amplitude $\phi(\boldsymbol{p})$. The interpolated outcome of the numerical integration is compared, in Fig. 3(a), with the simple parametrization, meaningful if $\eta>\frac{3}{4}$,

$$
\varphi_{2}(p)=\sqrt{\frac{\Gamma(2 \eta)}{\sqrt{\pi} \Gamma\left(2 \eta-\frac{3}{2}\right)}} \frac{2 s^{2 \eta-\frac{3}{2}}}{\left(p^{2}+s^{2}\right)^{\eta}}, \quad\left\|\varphi_{2}\right\|^{2} \equiv \int_{0}^{\infty} \mathrm{d} p p^{2}\left|\varphi_{2}(p)\right|^{2}=1,
$$

for the numerical values of the two parameters, $s$ and $\eta$, in Table 3 . In spite of the simplicity of this parametrization, it exhibits almost perfect agreement with the integration outcome.

Table 2: Numerical parameter values fixing our wave-function renormalization ansatz (14).

\begin{tabular}{lcccccc}
\hline \hline Parameter & $u$ & $v$ & $x\left[\mathrm{GeV}^{-1}\right]$ & $y\left[\mathrm{GeV}^{-2}\right]$ & $z\left[\mathrm{GeV}^{-4}\right]$ & $\delta$ \\
\hline Value & 1.01234 & 0.374334 & 0.229377 & 0.258882 & 0.592377 & 0.313777 \\
\hline \hline
\end{tabular}




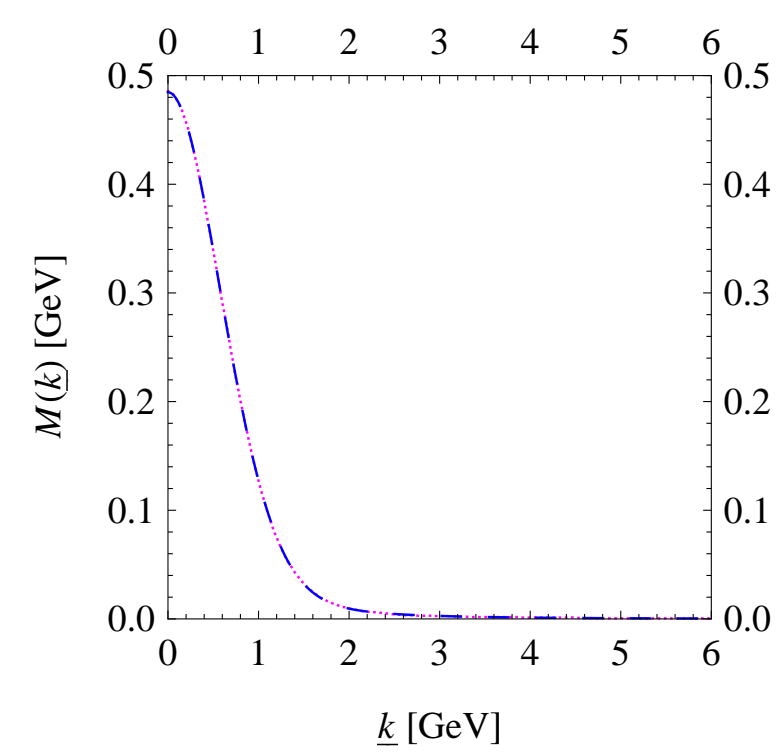

(a)

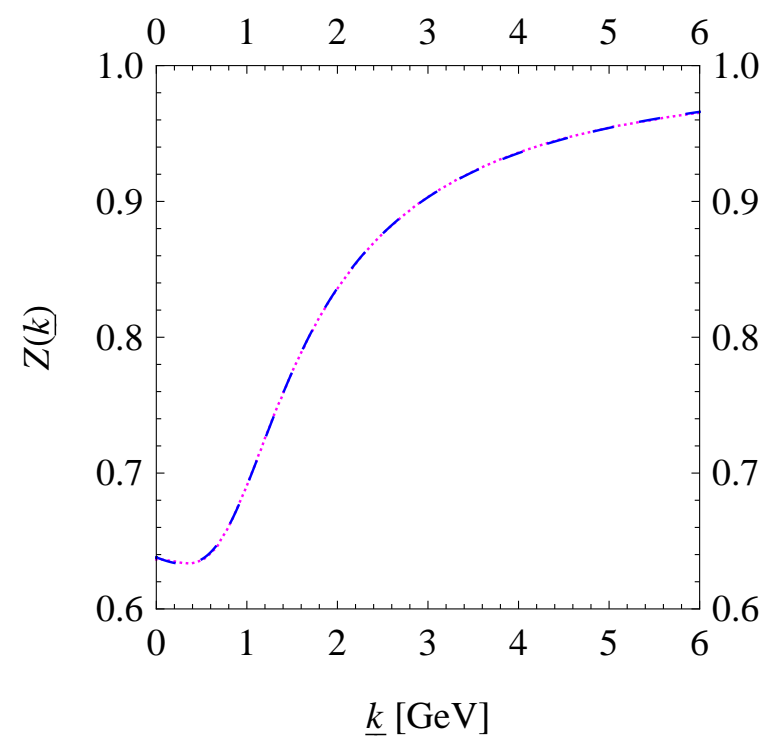

(b)

Figure 2: Comparison of (a) the parametrization (13) of the mass function $M(\underline{k})$ for the five parameters of Table 1 (blue dashed line) with its interpolation in Fig. 1(a) (magenta dotted line) and (b) the parametrization (14) of the wave-function renormalization $Z(\underline{k})$ for the fit parameters of Table 2 (blue dashed line) with its origins in Fig. 1(b) (magenta dotted line).

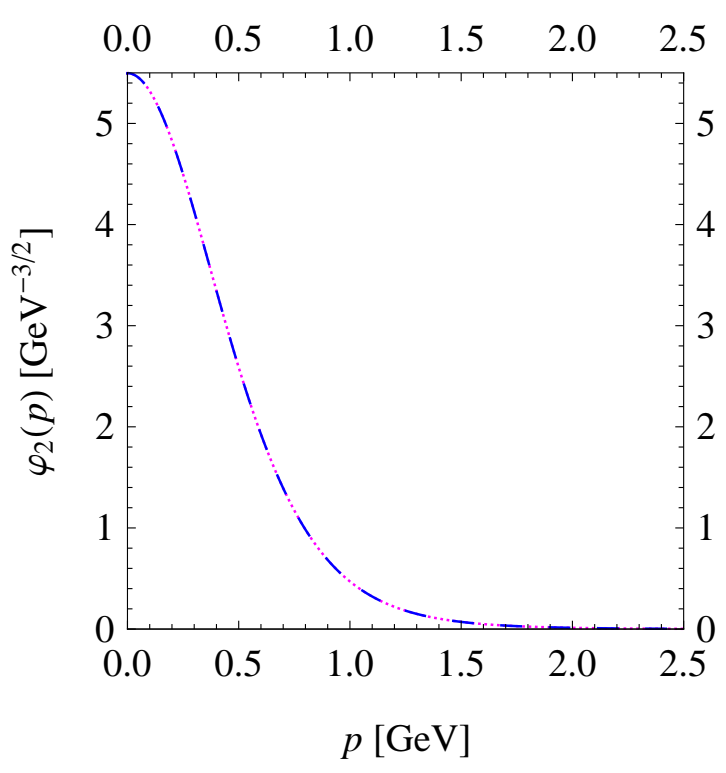

(a)

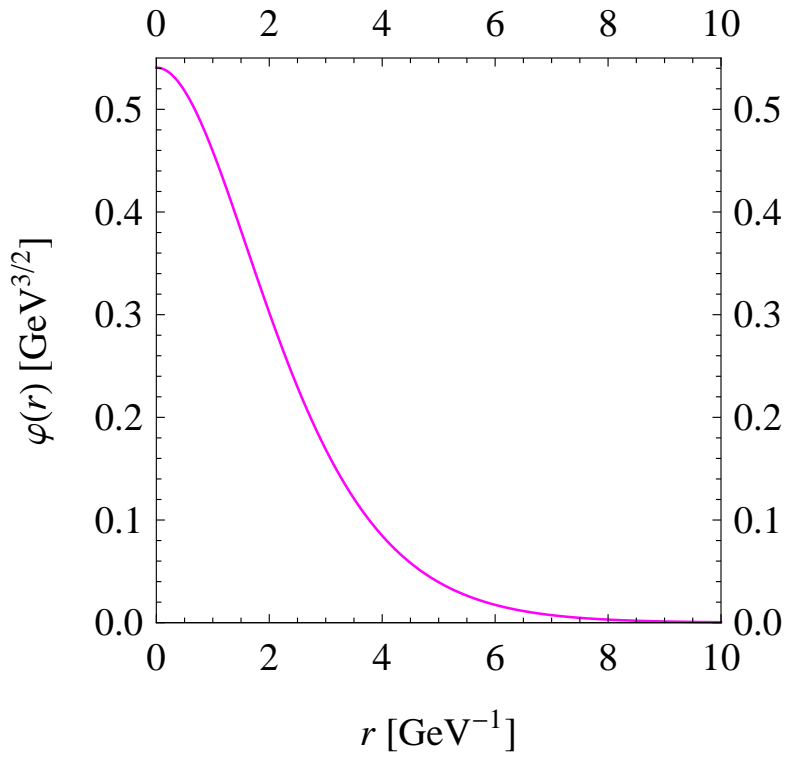

(b)

Figure 3: Radial Salpeter amplitude for Goldstone-type quark-antiquark bound states: (a) its momentum-space behaviour $\varphi_{2}(p)$, given by the outcome of the numerical integration of its Bethe-Salpeter amplitude (12) with respect to Euclidean time (magenta dotted line) or, equally well, the simple parametrization (15) (blue dashed line); (b) its configuration-space behaviour $\varphi(r)$, found as Fourier-Bessel transform of that ansatz (15) (magenta solid line). 
Table 3: Constants enabling us to reproduce the Salpeter function $\varphi_{2}(p)$ by our ansatz (15).

\begin{tabular}{lcc}
\hline \hline Parameter & $s[\mathrm{GeV}]$ & $\eta$ \\
\hline Value & 1.16176 & 4.43353 \\
\hline \hline
\end{tabular}

As our final move, we clearly have to switch over, by straightforward application of both Fourier-Bessel transformations (10), to configuration space. For the function $\varphi_{2}(p)$, thanks to the simplicity of our representation (15), this can be accomplished by analytic means. In terms of the modified Bessel functions of the second kind of order $\sigma \in \mathbb{R}[21], K_{\sigma}(z)$, we get

$$
\varphi(r)=\sqrt{\frac{\Gamma(2 \eta)}{\sqrt{\pi} \Gamma\left(2 \eta-\frac{3}{2}\right)}} \frac{2^{2-\eta} s^{\eta}}{\Gamma(\eta)} r^{\eta-\frac{3}{2}} K_{\frac{3}{2}-\eta}(s r), \quad\|\varphi\|^{2} \equiv \int_{0}^{\infty} \mathrm{d} r r^{2}|\varphi(r)|^{2}=1 .
$$

The behaviour of $\varphi(r)$, depicted in Fig. 3(b), does not offer any unexpected surprise. As far as the kinetic term is concerned, because of the presence of the quark propagator functions, $M\left(p^{2}\right)$ and $Z\left(p^{2}\right)$, the Fourier-Bessel transform $T(r)$ has to be computed numerically. This implies that for the underlying interaction potential $V(r)$ no analytic representation can be given. Figure 4 shows its dependence on the interquark distance $r$ as inferred from Eq. (11).

As a function of the interquark distance $r$, the configuration-space interaction potential $V(r)$ displayed in Fig. 4 exhibits a not extraordinarily spectacular behaviour: at the spatial origin $r=0$, it starts from its negative value $V(0)=-1.91772 \mathrm{GeV}$, then remains, without very much variation or modulation, below zero until it crosses the abscissa at its single zero at $r=15.6978 \mathrm{GeV}^{-1}$, in order to move on to an extremely steep monotonic rise to infinity, bearing a coarse resemblance to a smoothed infinite square well, and entailing confinement.

\section{Summary, Findings, Conclusions, and Perspectives}

The present investigation was devoted to the application of (the instantaneous limit of) the homogeneous Bethe-Salpeter formalism to the description of the pseudo-Goldstone bosons of the chiral symmetries of QCD, in an insignificantly idealized disguise, namely, as strictly massless mesons. In particular, we explored the respective capabilities of the instantaneous Bethe-Salpeter equation formulated in Ref. [2] that (in spite of the reduction it underwent) still has memories of sufficient intensity to its quantum-field-theoretic origins to encompass effects decisive for the understanding of the dynamical breakdown of the chiral symmetries.

We were able to show that this specific bound-state equation enables us to deal with the Goldstone bosons in a surprisingly simple manner: As illustrated by Fig. 4, the shape of the configuration-space potential introducing the strong interactions into the integral kernel of this equation of motion proves to be pretty close to a (bag-model-type) infinite square well. In contrast to that, in our previous analyses of similar applications of the Salpeter equation we encountered parameter-dependent occurrences of singularities or nonconfinement [6-8].

Moreover, in these earlier works we ignored the implications of the quark wave-function renormalization factors, either because we merely exploited one or the other specific aspect of the quark mass functions $[6,7]$ or simply because of the lack of availability of appropriate 


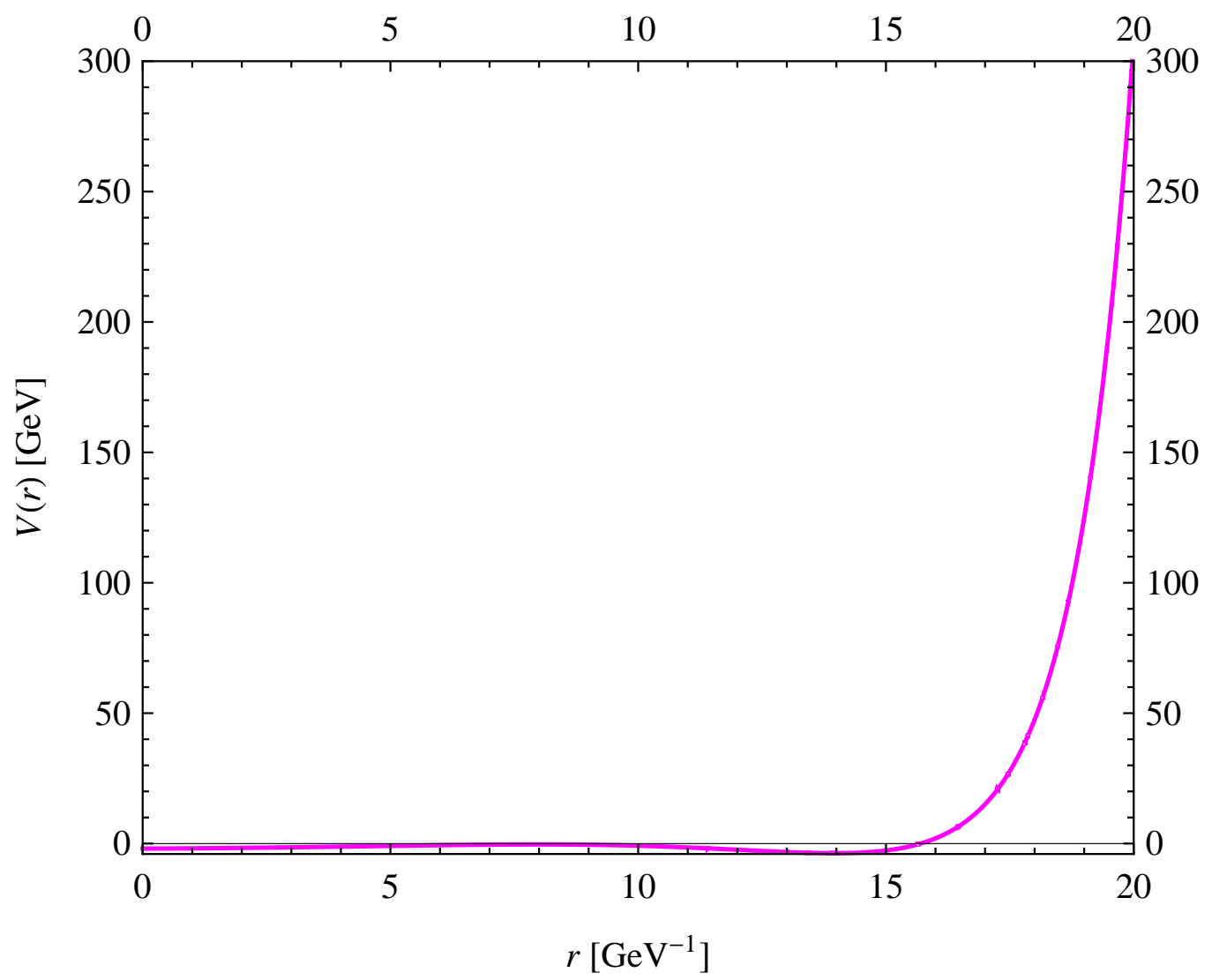

Figure 4: Configuration-space potential $V(r)$ derived by inverting the bound-state problem posed by the ("Salpeter-approach-improving") instantaneous Bethe-Salpeter equation (1) for fermion-antifermion bound states proposed in Ref. [2], with the Fierz-invariant Lorentz structure (7) of its interaction kernel, upon application of our (Ward-Takahashi-mediated) knowledge of its solutions describing nearly massless Goldstone-type pseudoscalar mesons.

results in the literature for the wave-function renormalization function from the underlying Dyson-Schwinger truncation model [8]. Here, the quark wave-function renormalization has been taken into account, which entails that the relationship between quark propagator and pseudoscalar-meson Bethe-Salpeter amplitude has been used more accurately than before. A closer inspection reveals that this move has discernible quantitative consequences for the behaviour of the potential but will hardly imply a dramatic qualitative impact on its shape.

Of these insights clearly most essential is the square-well shape of the potential. ${ }^{5}$

Finally, a word of caution: By a too cursory interpretation of the inversion outcomes for the interquark potential $V(r)$ shown in Fig. 4, one might naïvely suspect that this potential should describe mesonic bound states that are much larger than what one would expect for the size of, e.g., the pion. However, already by construction the ground-state solution of the Bethe-Salpeter quintessence (9) with the interquark potential $V(r)$ of Fig. 4 is the Salpeter component wave function $\varphi(r)$ that provides the starting point of our inversion adventures.

\footnotetext{
${ }^{5}$ Needless to say, in this context we use the notion "square well" just as a metaphor for the $r$ dependence of the interquark potential $V(r)$ observed in Sec. 5, namely, a rather flat behaviour near the origin $r=0$ up to a critical distance beyond which, for $r \rightarrow \infty$, an extremely steep rise, with finite slope, to infinity follows.
} 
(The latter claim can be easily verified by, e.g., application of some variational techniques.) Realizing this, from a brief inspection of the behaviour of $\varphi(r)$ given in Fig. 3(b), one learns that the corresponding hadronic extension should be of the order of some $2.5 \mathrm{GeV}^{-1}$. More precisely, our $\varphi(r)$ - and thus the bound-state equation (9) with interquark potential $V(r)$ as in Fig. 4 - describes massless mesons of average interquark distance $\langle r\rangle=0.483 \mathrm{fm}$ and root-mean-square radius $\sqrt{\left\langle r^{2}\right\rangle}=0.535 \mathrm{fm}$. Confronting our results with the experimental

outcomes [22] for the electromagnetic charge radius of the pion $\sqrt{\left\langle r_{\pi}^{2}\right\rangle}=(0.672 \pm 0.008) \mathrm{fm}$, we find a reasonable proximity of the numerical findings, lending credibility to our analysis.

\section{References}

[1] H. A. Bethe and E. E. Salpeter, Phys. Rev. 82 (1951) 309;

M. Gell-Mann and F. Low, Phys. Rev. 84 (1951) 350;

E. E. Salpeter and H. A. Bethe, Phys. Rev. 84 (1951) 1232.

[2] W. Lucha and F. F. Schöberl, J. Phys. G: Nucl. Part. Phys. 31 (2005) 1133, arXiv:hepth/0507281.

[3] E. E. Salpeter, Phys. Rev. 87 (1952) 328.

[4] W. Lucha and F. F. Schöberl, Phys. Rev. D 87 (2013) 016009, arXiv:1211.4716 [hep-ph].

[5] W. Lucha, Proc. Sci., EPS-HEP 2013 (2013) 007, arXiv:1308.3130 [hep-ph].

[6] W. Lucha and F. F. Schöberl, Phys. Rev. D 92 (2015) 076005, arXiv:1508.02951 [hep-ph].

[7] W. Lucha and F. F. Schöberl, Phys. Rev. D 93 (2016) 056006, arXiv:1602.02356 [hep-ph].

[8] W. Lucha and F. F. Schöberl, Phys. Rev. D 93 (2016) 096005, arXiv:1603.08745 [hep-ph].

[9] W. Lucha and F. F. Schöberl, Int. J. Mod. Phys. A 07 (1992) 6431.

[10] W. Lucha, F. F. Schöberl, and D. Gromes, Phys. Rep. 200 (1991) 127.

[11] Li Z.-F., W. Lucha, and F. F. Schöberl, Mod. Phys. Lett. A 21 (2006) 1657, arXiv:hep$\mathrm{ph} / 0510372$.

[12] W. Lucha and F. F. Schöberl, in Quark Confinement and the Hadron Spectrum VII: $7^{\text {th }}$ Conference on Quark Confinement and the Hadron Spectrum - QCHST, edited by J. E. F. T. Ribeiro, AIP Conf. Proc. (AIP, Melville, New York, 2007), Vol. 892, p. 524, arXiv:hep$\mathrm{ph} / 0610016$.

[13] W. Lucha, K. Maung Maung, and F. F. Schöberl, Phys. Rev. D 63 (2001) 056002, arXiv:hep$\mathrm{ph} / 0009185$.

[14] W. Lucha, K. Maung Maung, and F. F. Schöberl, in Proceedings of the International Conference on Quark Confinement and the Hadron Spectrum IV, edited by W. Lucha and K. Maung Maung (World Scientific, Singapore, 2002), p. 340, arXiv:hep-ph/0010078.

[15] W. Lucha, K. Maung Maung, and F. F. Schöberl, Phys. Rev. D 64 (2001) 036007, arXiv:hep$\mathrm{ph} / 0011235$.

[16] W. Lucha and F. F. Schöberl, Int. J. Mod. Phys. A 17 (2002) 2233, arXiv:hep-ph/0109165. 
[17] J.-F. Lagaë, Phys. Rev. D 45 (1992) 305;

M. G. Olsson, S. Veseli, and K. Williams, Phys. Rev. D 52 (1995) 5141, arXiv:hep$\mathrm{ph} / 9503477$.

[18] P. Maris, C. D. Roberts, and P. C. Tandy, Phys. Lett. B 420 (1998) 267, arXiv:nuclth/9707003.

[19] P. Maris and P. C. Tandy, Phys. Rev. C 60 (1999) 055214, arXiv:nucl-th/9905056.

[20] P. Maris, in Proceedings of the International Conference on Quark Confinement and the Hadron Spectrum IV, edited by W. Lucha and K. Maung Maung (World Scientific, Singapore, 2002), p. 163, arXiv:nucl-th/0009064.

[21] Handbook of Mathematical Functions, edited by M. Abramowitz and I. A. Stegun (Dover, New York, 1964).

[22] C. Patrignani et al. (Particle Data Group), Chin. Phys. C 40 (2016) 100001. 